\title{
Pitting Corrosion Morphology Characteristics of 7B04 Aluminum Alloys In Airport Environment Based on Fractal Theory
}

\author{
Zhi-guo LIU ${ }^{1, a, *}$, Na ZHANG ${ }^{1, b}$ and Guang-yao YAN ${ }^{1, c}$ \\ ${ }^{1}$ Naval Aeronautical Engineering Academy Qingdao Branch, Qingdao, China \\ a lqdnuaalzg@163.com, b35517664@qq.com, cxdli23615046@163.com
}

Keywords: Aluminum alloys, Pitting corrosion, Morphology characteristics, Fractal theory, Fractal dimension

\begin{abstract}
Aircraft aluminum alloy is easy to initiate pitting corrosion in the airport environment, and the pitting corrosion topography characteristics could directly affect the fatigue mechanical property of aluminum alloy structure. In order to obtain the pitting corrosion characteristics of 7B04 aluminum alloy in the airport environment, the pitting corrosion test was carried out according to the accelerated corrosion environment spectrum which simulated the airport environment, and the corrosion damage parameters were defined, including corrosion pit depth $H$, corrosion pit surface length $L$ and the corrosion pit surface width $W$. During the corrosion test process, the three parameters of typical corrosion pits were successively measured in different corrosion period to obtain the corrosion pit damage size data, then the data was statistically analysed and used to compute the fractal dimension of corrosion damage based on the fractal theory by which to gain the pitting corrosion damage characteristics and its evolvement rules during pitting corrosion process. The computation results show that the corrosion pit damage clearly appears fractal characteristics and the fractal dimension of corrosion pit gradually increases with corrosion time prolongation, which suggests that the corrosion pit damage trends to be shallow and long and moderate wide topography character in the later corrosion period.
\end{abstract}

\section{Introduction}

Aircraft aluminum alloy is susceptible to pitting corrosion in the environment during service process, and corrosion pit damage is easy to nucleate fatigue crack when it is subjected to fatigue loading spectrum which could drastly shorten the fatigue life and affect the integrity of structure[1-2], so the behaviour of pitting corrosion initiation and growth is of vital importance to the corrosion fatigue analysis of aircraft aluminum alloy structure, especially the pitting corrosion damage topography characteristics, which could directedly affect the degeneration degree and degeneration probability of aluminum structure fatigue property[3-4]. From this view, the pitting corrosion damage topography and its relevant context are regarded as the foundation of corrosion fatigue life research of aircraft structure. In this paper, 7B04 aluminum alloy is chosen as research object, and pitting corrosion is viewed as a three-dimensional flaw which can be depicted by the depth and the surface length and the surface width of corrosion pit, and the fractal theory method is adopted to analyse the corrosion pit damage topography characteristics based on the 7B04 specimen pitting corrosion test result.

\section{The Pitting Corrosion Test and Test Data of 7B04 Specimen}

The pitting corrosion test of 7B04 specimen was carried out according to environment spectrum which simulated the aircraft service environment and was depicted in literature[5] detailedly. The corrosion pit damage parameters were defined as following to describe the corrosion damage topography: firstly, the corrosion pit depth was the distance between the specimen surface and pit bottom which represented by parameter $H$, secondly, the corrosion pit length was pit surface length parallel to the axial orientation of specimen which represented by parameter $L$, finally, the 
corrosion pit width was the pit surface width vertical to the axial orientation of specimen which represented by parameter $W$.The unit of parameters was $\mu m$, and their definition was shown in Fig.1.

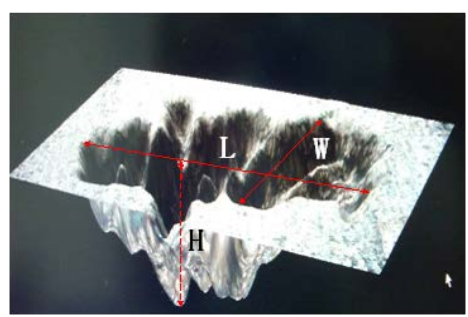

Figure 1 Schematic diagram of corrosion pit topography parameter.

The accelerated corrosion test lasted 13 equivalent corrosion years[5], and typical macroscopic corrosion topography of 7B04 samples in different corrosion period was shown in Fig.2.

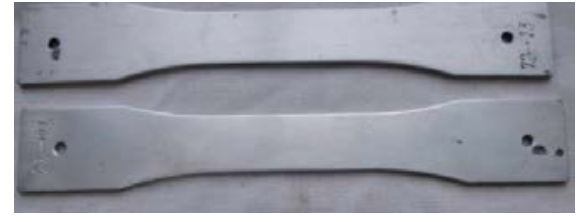

(a) $3 \mathrm{a}$

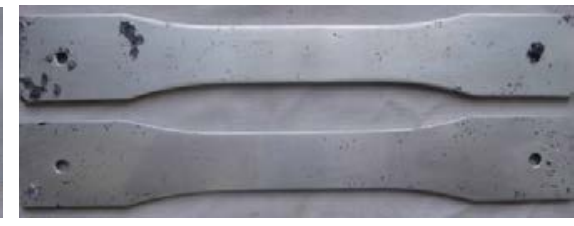

(b) $5 \mathrm{a}$

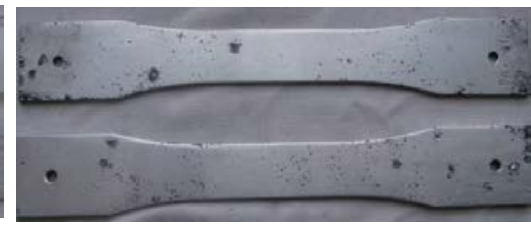

(c) $10 \mathrm{a}$

Figure 2 Pitting corrosion macroscopic topography of 7B04 samples in different corrosion year.

Typical corrosion pit damage parameters were successively measured through three-dimensional microscope during the corrosion test between a fixed certain interval, and part of the whole pit damage parameters data were shown in Table 1

Table 1 The corrosion pit topography parameters of 7B04 specimen in different corrosion year.

\begin{tabular}{ccccc}
\hline Corrosion year/a & 5 & 7 & 9 & 11 \\
\hline & $55.0 \times 26.0 \times 0.8$ & $60.0 \times 20.0 \times 1.5$ & $90.0 \times 20.0 \times 1.7$ & $150.0 \times 35.0 \times 1.8$ \\
& $41.0 \times 20.0 \times 0.6$ & $95.0 \times 21.0 \times 1.2$ & $96.0 \times 25.0 \times 1.7$ & $120.0 \times 33.0 \times 1.9$ \\
$L \times W \times H$ & $40.0 \times 16.0 \times 0.6$ & $55.0 \times 18.0 \times 0.7$ & $110.0 \times 20.0 \times 1.5$ & $120.0 \times 34.0 \times 2.1$ \\
$\left(\mu m^{3}\right)$ & $\ldots \ldots \ldots \ldots$ & $\ldots \ldots \ldots \ldots$ & $\ldots \ldots \ldots$. & $\ldots \ldots \ldots \ldots$ \\
& $80.0 \times 12.0 \times 0.8$ & $83.0 \times 26.0 \times 1.5$ & $90.0 \times 30.0 \times 1.8$ & $122.0 \times 33.0 \times 2.0$ \\
& $41.0 \times 18.0 \times 0.7$ & $60.0 \times 21.0 \times 0.8$ & $60.0 \times 30.0 \times 1.5$ & $89.0 \times 35.0 \times 1.9$ \\
& $44.0 \times 22.0 \times 1.0$ & $56.0 \times 24.0 \times 1.1$ & $85.0 \times 27.0 \times 1.2$ & $173.0 \times 36.0 \times 2.5$ \\
\hline
\end{tabular}

\section{Fractal Theory}

Pitting corrosion of aluminum alloy is a nonlinear process in essence which is suitable for being analysed by the nonlinear science and method[6-7]. The fractal theory is adopted here to modelling the pitting corrosion topography evolvement because of its capability to solving the nonlinear problem.

The fractal theory is one of modern nonlinear science whose key core is the fractal dimension, according to different research subject, the fractal dimension has different definition ways, including the resemblance dimension, Hausdorff dimension, box dimension, spectrum dimension and topology dimension and so on[8].

The Hausdorff dimension was used here to handle the pitting corrosion test data of 7B04 specimen which were shown in Table 1.The principle of the Hausdorff dimension is as following: set $\varepsilon$ as the benchmark unit, then get the contour scale $N$, so there is a function relationship between $\varepsilon$ and $N$ which could be expressed with $N(\varepsilon)$, and the smaller $\varepsilon$, the larger $N$, so the relationship could be expressed with power function which is shown in equation (1).

$$
N(\varepsilon) \propto \varepsilon^{-D}
$$


In equation (1), the letter $D$ represents the Hausdorff fractal dimension, and its value could be calculated from the following expression (2).

$$
D=\frac{\ln N\left(\varepsilon_{2}\right)-\ln N\left(\varepsilon_{1}\right)}{-\left(\ln \varepsilon_{2}-\ln \varepsilon_{1}\right)}
$$

\section{Fractal Dimension Computation of Pitting Corrosion Damage}

Test data of typical corrosion period which included 5, 9 and 11 equivalent corrosion year were chosen to computation the fractal dimension value according to the fractal theory and equation (2).

First of all, the pit depth parameter $H$ was chose as an analysis example. In order to compute the Hausdorff fractal dimension of parameter $H$, the corrosion pit data in above mentioned corrosion period were statistically analysed and got the typical $H$ value and its corresponding frequency in different equivalent corrosion year, then the value and the frequency were dealt with with logarithm transformation ways through which the scatter diagram and the fitting line could be got, the scatter diagram and the fitting line of typical corrosion pit depth $H$ in different corrosion period were shown in Fig.3, according to equation (2), we could conclude that the slope of fitting line was the Hausdorff fractal dimension $D$ of corrosion pit depth.
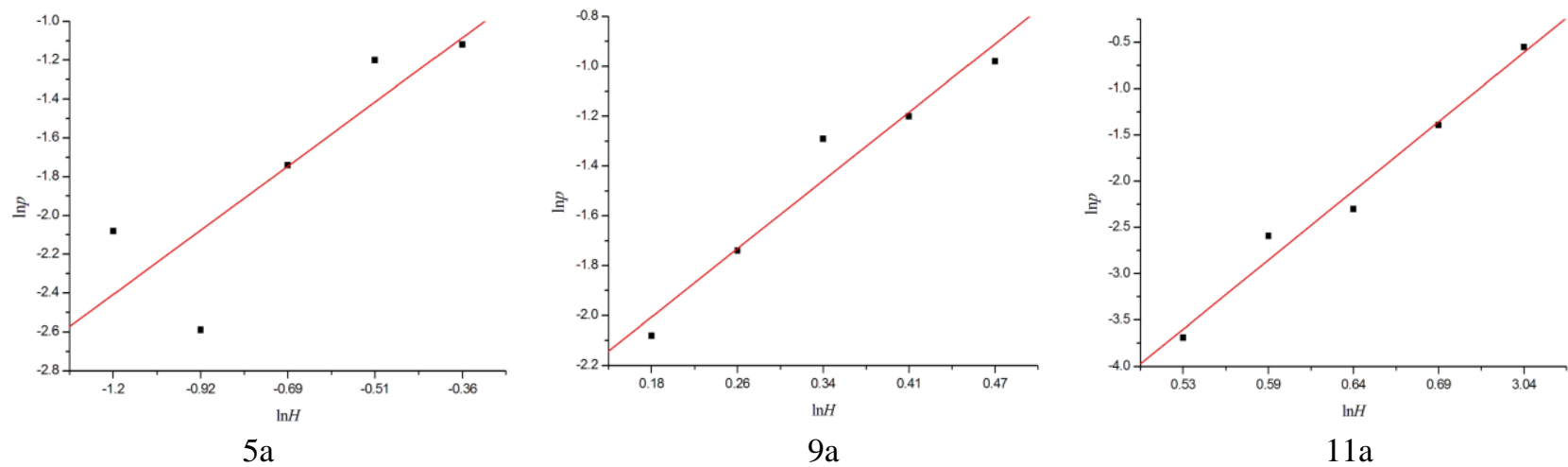

Figure 3 The fractal characteristics of corrosion pit depth in different corrosion period.

Similarly to the above mentioned method, the the Hausdorff fractal dimension $D$ of corrosion pit surface length $L$ in different corrosion period also could be obtained which were shown in Fig.4.The fractal characteristics of corrosion pit surface width $W$ was not listed because of similarity.
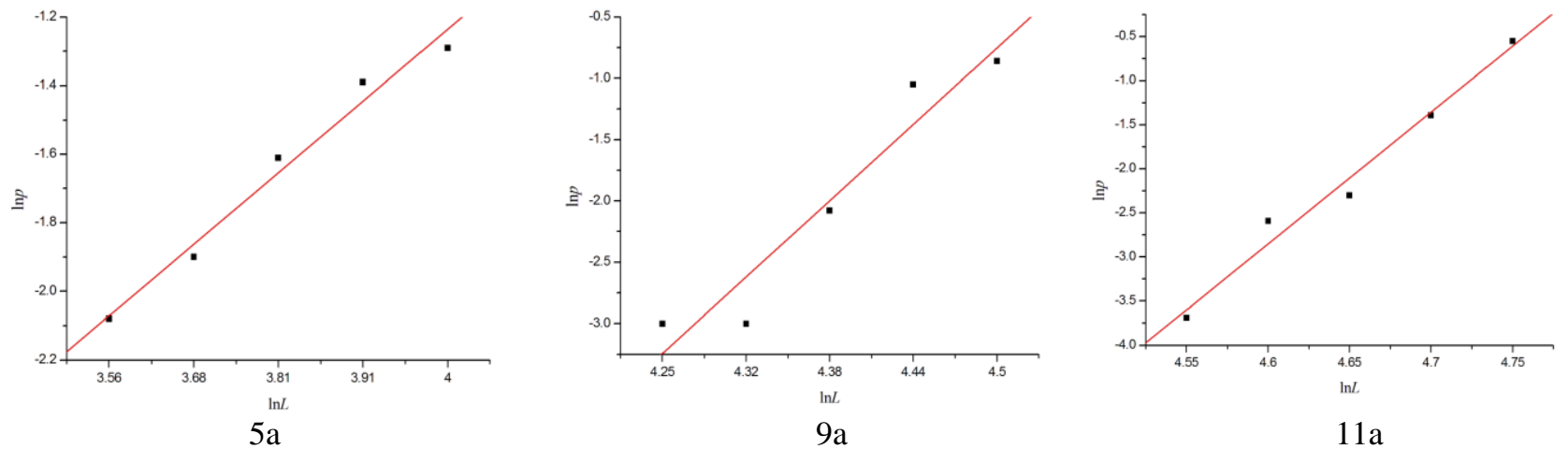

Figure 4 The fractal characteristics of corrosion pit surface length in different corrosion period.

\section{Discussion}

According to the computation result and Fig.3 and Fig.4,it is obviously that the corrosion pit damage of 7B04 specimen has fractal characteristics and the fractal dimension value of corrosion 
damage increases with the corrosion period prolongation. Based on the physical meaning and evolvement rules of fractal dimension, we can conclude that the values pitting corrosion damage parameters have larger randomness in the former corrosion period and gradually inclines to the stable tendency respectively in the later corrosion period.

The conclusion is consistent with the physical process of aluminum alloy pitting corrosion. Literature [9-10] regards the pitting corrosion behaviour of aluminum alloy as a metastable process in essence in the initial corrosion period which has an intrinsic stochastic nature and is affected by many factors[11-12],so the values of corrosion pit damage parameters, including the pit depth $H$ and pit surface length $L$ and surface width $W$, are of randomness character in the former corrosion period which causes the smaller value of Hausdorff fractal dimension $D$, then the pitting corrosion behaviour gradually transforms from metastable status to stable event, during the process ,the rate of new corrosion pit initiation gradually decreases and the former existent corrosion pits incline to the stable growth which makes the values of corrosion pits damage evenly. This uniformed character of corrosion pit damage in the middle and later corrosion stage make the Hausdorff fractal dimension $D$ of parameter $H$ and $L W$ gradually increase, and the fractal characteristics of corrosion pit damage becomes more and more obvious.

Based on the above-mentioned discussion and the data shown in Table 1,the conclusion can be made that the pitting corrosion damage topography has fractal character which is more obvious with the corrosion period increment, and the values of corrosion pit damage parameters trend to individual extremum, that is to say, the depth value of most corrosion pits inclines to smaller value, and the surface length and width value of most corrosion pits incline to larger value and middle value respectively. In one word, the corrosion pit topography of 7B04 aluminum alloy wholly has the characteristics of relatively shallow in depth and longer in surface length and middle width in surface width, which is as shown in Fig.5.

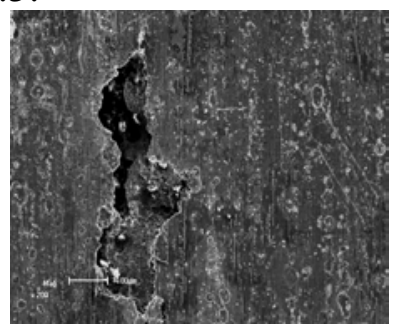

Figure 5 Typical corrosion pit topography SEM of specimen (11 corrosion year).

\section{Conclusion}

Accelerated corrosion test of 7B04 specimen was carried out according to the corrosion environment spectrum, and test data of typical corrosion pits was measured and processed through the fractal theory to obtain the fractal character of 7B04 aluminum alloy pitting corrosion damage topography in service environment. The research results find that:

(1) The corrosion pit damage of 7B04 aluminum alloy has obvious fractal characteristics and the fractal dimension value of corrosion damage increases with the corrosion period prolongation.

(2) The corrosion pit damage topography of 7B04 aluminum alloy has the characteristics of relatively shallow in depth and longer in surface length and middle width in surface width in the later corrosion period.

\section{References}

[1] Maganioti, A.E., Chrissanthi, H.D., Charalabos, P.C., Andreas, R.D., George, P.N. and Christos, C.N. (2010) Cointegration of Event-Related Potential (ERP) Signals in Experiments with Different Electromagnetic Field (EMF) Conditions. Health, 2, 400-406.

[2] Bootorabi, F., Haapasalo, J., Smith, E., Haapasalo, H. and Parkkila, S. (2011) Carbonic Anhydrase VII—A Potential Prognostic Marker in Gliomas. Health, 3, 6-12. 
[1] Pan Shi, Sankaran Mahadevan.Damage tolerance approach for probabilistic pitting corrosion fatigue life prediction[J].Engineering Fracture Mechanics, 68(2001):1493-1507.

[2]K.K. Sankaran, R. Perez , K.V. Jata. Effects of pitting corrosion on the fatigue behavior of aluminum alloy 7075-T6: modeling and experimental studies[J]. Materials Science and Engineering ,A297 (2001): 223-229.

[3] ZHANG Chuan.Research on Residual Life and Residual Strength of Metals with Corrosion Damage[D].Nanjing: Nanjing University of Aeronautics and Astronautics,2012.

[4] LI Fumin, YUAN Yingshu. Distributing character of size of pits on steel strands corroded by chloride[J]. JOURNAL OF CHINA COAL SOCIETY, 2011, 36(11):1826-1831.

[5] LIU Zhiguo, MU Zhitao, BIAN Ruopeng. STUDY ON ARIMA MODEL OF LD2 ALUMINUM ALLOY ACCELERATED CORROSION PIT EVOLVEMENT[J]. Journal of Mechanical Strength,2012,34(4):608-614.

[6] Wei Robert P. A model for particle-induced pit growth in aluminum alloys[J]. Scripta Mater, 2001,44:2647-2652.

[7] D. Gary Harlow, Robert P. Wei. Probability modeling and material microstructure applied to corrosion and fatigue of aluminum and steel alloys[J].Engineering Fracture Mechanics,76 (2009) :695-708.

[8] Mandelbrot B.B.,CHEN Shouji, LING Fuhua.The Fractal Geometry of Nature[M]. Shanghai: Shanghai Far-east Press,1998.

[9] N. Murer, R.G. Buchheit, Stochastic modelling of pitting corrosion in aluminum alloys[J]. Corrosion Science, 69 (2013) 139-148.

[10] A.Valor, F. Caleyo, L. Alfonso, D. Rivas, J.M. Hallen. Stochastic modeling of pitting corrosion: A new model for initiation and growth of multiple corrosion pits[J]. Corrosion Science, 49 (2007) 559-579.

[11] K. van der Walde, B.M. Hillberry. Initiation and shape development of corrosion-nucleated fatigue cracking[J]. International Journal of Fatigue, 29 (2007):1269-1281.

[12] D.G.Harlow, J.Nardiello, J. Payne. The effect of constituent particles in aluminum alloys on fatigue damage evolution: Statistical observations[J]. International Journal of Fatigue, 32 (2010): 505-511. 